M. Andreatta and G. Occhetta

Nagoya Math. J.

Vol. 168 (2002), 127-137

\title{
SPECIAL RAYS IN THE MORI CONE OF A PROJECTIVE VARIETY
}

\section{MARCO ANDREATTA AND GIANLUCA OCCHETTA}

\begin{abstract}
Let $X$ be a smooth $n$-dimensional projective variety over an algebraically closed field $k$ such that $K_{X}$ is not nef. We give a characterization of non nef extremal rays of $X$ of maximal length (i.e of length $n-1$ ); in the case of $\operatorname{Char}(k)=0$ we also characterize non nef rays of length $n-2$.
\end{abstract}

\section{$\S 1$. Introduction}

Let $X$ be a smooth $n$-dimensional projective variety over an algebraically closed field $k$ of arbitrary characteristic.

We assume that $K_{X}$ is not nef, in particular that there exists an $e x$ tremal ray $R$ in the cone $\overline{N E(X)}_{K_{X}<0}$.

The length of the ray $R$ is the integer defined as $l(R)=\min \left\{-K_{X} \cdot C\right.$ : $[C] \in R\}$; the set $\operatorname{Locus}(R)$ is the set of closed point $x \in X$ such that there is a curve $x \in C^{\prime} \subset X$ with $\left[C^{\prime}\right] \in R$.

A ray $R$ is said to be nef if $D . R \geq 0$ for all effective divisors $D \subset X$, or equivalently if $\operatorname{Locus}(R)=X$; if a nef ray exists the variety $X$ is therefore uniruled.

The main result of the paper is the following characterization of the blow-up of a smooth point in terms of extremal rays.

THEOREM 1.1. Let $X$ be a smooth n-dimensional projective variety. There exists a non nef extremal ray $R$ of length $\geq(n-1)$ if and only if there exists a morphism $\varphi: X \rightarrow X^{\prime}$ into a smooth projective variety $X^{\prime}$ which is the blow-up of $X^{\prime}$ at a point.

Let us point out that in the case $\operatorname{Char}(k)>0$ the existence of a map $\varphi: X \rightarrow X^{\prime}$ which contracts all the curves in an extremal ray has not been proved in general; if Char $(k)=0$ this is the so called Kawamata-Shokurov contraction theorem.

Received October 6, 2000.

2000 Mathematics Subject Classification: Primary 14E30; Secondary 14J40. 
A straightforward application of this result is in the so called adjunction theory: namely if $L$ is an ample line bundle on a smooth $n$-dimensional projective variety $X$ one can study the ampleness of the adjoint bundle $K_{X}+(n-1) L$ : this is ample unless there exist extremal rays of length $\geq(n-1)$.

If these rays are nef they will determine the uniruled variety $X$; this is the case if $l(R)>(n-1)$, see [KK00], or if $\operatorname{Char}(k)=0$, see [Fuj87]; if $l(R)=n-1$ the precise characterization of $X$ is still open in positive characteristic (see Remark (4.3)).

If there are non nef rays one can apply the above theorem and the following happens:

THEOREM 1.2. Let $X$ be a smooth $n$-dimensional projective variety with $n \geq 3$ and $L$ an ample line bundle on $X$.

If there is a non nef ray in the cone $\overline{N E(X)} \overline{(K X}_{X+(n-1) L) \leq 0}$ then, with the only exception given by the blow-up of $\mathbb{P}^{3}$ in one point, $\pi: B l_{x} \mathbb{P}^{3} \rightarrow \mathbb{P}^{3}$, and $L=\pi^{*}\left(\mathcal{O}_{\mathbb{P}^{3}}(2)\right)-\left[\pi^{-1}(x)\right]$, all rays in this cone are non nef and they can be simultaneously contracted into a smooth variety $X^{\prime}$, with a morphism $\varphi: X \rightarrow X^{\prime}$ expressing $X$ as blow-up of $X^{\prime}$ at a finite set of points $B$.

Moreover there is an ample line bundle $L^{\prime}$ on $X^{\prime}$ such that $L \otimes\left(\left[\varphi^{-1}(B)\right]\right)$ $=\varphi^{*} L^{\prime}$ and $K_{X^{\prime}}+(n-1) L^{\prime}$ is ample.

A similar statement holds for the case $n=2$ where extra care is needed for conic bundles. Actually the adjunction theory and the above theorem in case of surfaces were developed exactly a century ago in a paper of Castelnuovo and Enriques ([CE01], Firenze, fall 1900).

The above theorem was proved in the case $\operatorname{Char}(k)=0$ by A. Sommese who called the map $\varphi: X \rightarrow X^{\prime}$ the first reduction of $(X, L)$ (see [BS95] for a more general account as well as for applications of adjunction theory).

We will actually prove a more general version of the theorem (see (4.1)), where we consider a rank $(n-1)$ ample vector bundle $\mathcal{E}$ and we study the non nef rays in the cone $\overline{N E(X)}\left(K_{X}+\operatorname{det}(\mathcal{E})\right) \leq 0$ (this version of the theorem if $\operatorname{Char}(k)=0$ was proved in [ABW92]).

In Section 5 we restrict to the case $\operatorname{Char}(k)=0$; here we first generalize theorem (1.1) with a characterization of blow-up of smooth subvarieties in term of extremal rays (see 5.1). In particular we prove a conjecture stated in [AW97] for extremal rays whose associated contraction is divisorial.

Then we characterize non nef extremal rays of length $(n-2)$ (see $(5.2))$. In 
particular this result generalizes the theorem which guarantees the existence of the second reduction of a pair $(X, L)$ in the language of [BS95, Section 7.5].

The proof of our main theorem is strongly based on a recent result by Y. Kachi and J. Kollár [KK00]; we report this result in Section 2.

\section{§2. Set-up and preliminaries}

In this paper we will constantly use the notations and the definitions presented in the book of János Kollár, [Kol96], to which we will frequently refer. For the reader's convenience we recall here the principal ones.

Let $X$ be a smooth $n$-dimensional projective variety over an algebraically closed field of arbitrary characteristic. A rational curve on $X$ is an irreducible reduced curve $C \subset X$ whose normalization is $f: \mathbb{P}^{1} \rightarrow C \subset X$.

As we said in the introduction we assume that $K_{X}$ is not nef, in particular that there exists an extremal ray $R=\mathbb{R}^{+}[C]$ in the cone $\overline{N E(X)} K_{X}<0$ (see [Kol96, III.1.2]). We will choose the curve $C$ generating the ray to be rational and minimal with respect to the intersection with $-K_{X}$; sometimes we will say that such a $C$ is a minimal extremal rational curve. For such a curve $C$ the integer $l(R)=-K_{X} . C$ is called the length of the ray $R$.

We will denote by $V \subset \operatorname{Hom}_{\text {bir }}\left(\mathbb{P}^{1}, X\right)$ a closed irreducible subvariety, which is closed under $\operatorname{Aut}\left(\mathbb{P}^{1}\right)$ and which contains $C$; by the minimality of $C$ we have that $V$ is an unsplit family of rational curves (see [Kol96, IV.2]). We can define $\operatorname{Locus}(V)$ to be the image in $X$ of the natural morphism associated to the family of rational curves $V$ (see [Kol96, II.2.3]) and $\operatorname{Locus}(V, 0 \rightarrow x)$ to be the image of $V_{x}:=\{f \in V: f(0)=x\}$; since $V$ is unsplit $\operatorname{Locus}(V)$ is a proper closed subscheme.

We have the following fundamental inequality

Proposition 2.1. ([Kol96, IV.2.5 and 2.6.]) In the above notation and for a general $x \in \operatorname{Locus}(V)$ we have

$\operatorname{dim} \operatorname{Locus}(V)+\operatorname{dim} \operatorname{Locus}(V, 0 \rightarrow x)+1=\operatorname{dim} V \geq \operatorname{dim} X+\operatorname{deg}_{\left(-K_{X}\right)}(V)$.

We stress the fact that the equality holds since $V$ is an unsplit family. The formulation and the proof of this inequality is due to P. Ionescu and J. Wiśniewski, coming out from the fundamental construction of S. Mori.

One can prove (see [Kol96, III.1.4]) that, given an extremal ray $R$ on $X$, there exists a Cartier divisor $H_{R}$ on $X$ such that 
1. $H_{R}$ is nef.

2. If $z \in \overline{N E(X)}$ then $H_{R} . z=0$ iff $z \in R$.

Such an $H$ is called a supporting divisor for the extremal ray $R$.

Proposition 2.2. ([Kol96, III.1.6]) In the above notation one of the following two possibilities can occur

1. $\left(H_{R}\right)^{\operatorname{dim} X}>0$ iff there is an irreducible divisor $E \subset X$ such that $R . E<0$. In particular $\operatorname{Locus}(R) \subset E$; in this case we say that the ray $R$ is not nef.

2. $\left(H_{R}\right)^{\operatorname{dim} X}=0$ iff $\operatorname{Locus}(R)=X$ (thus $X$ is uniruled). In this case we say that the ray $R$ is nef.

Finally we recall the main result of the paper [KK00] which we will use in the proof of 1.1 :

Proposition 2.3. ([KK00, Lemma 8]) Let $X$ be a normal projective variety and $x \in X$ a smooth point. Let $H$ be an ample Cartier divisor on $X$ and let $\left\{C_{t}: t \in T\right\}$ be an $(n-1)$-dimensional family of curves through $x$. Assume that $\left(C_{t} \cdot H\right)=1$ and $\left(-K_{X} \cdot H^{n-1}\right)>(n-1)\left(H^{n}\right)$. Then $X \cong \mathbb{P}^{n}$, $H=\mathcal{O}_{\mathbb{P} n}(1)$ and the $C_{t}$ are lines through $x$.

\section{§3. Non nef extremal rays with maximal length}

In this section we prove the main theorem (1.1) stated in the introduction.

The if part of the theorem is trivial.

Assume therefore that $R$ is a non nef extremal ray of length $\geq(n-1)$; thus by (2.2) there exists an irreducible divisor $E$ such that $\operatorname{Locus}(R)$ is contained in $E$ and E.R $<$.

Let $V$ be an unsplit family of deformations of $C$, a minimal extremal rational curve generating the ray and let $x$ be a general point in $\operatorname{Locus}(V)$; applying the inequality in (2.1) we obtain that

$$
\operatorname{dim} \operatorname{Locus}(V)+\operatorname{dim} \operatorname{Locus}(V, 0 \rightarrow x)+1=\operatorname{dim} V \geq 2 n-1 .
$$

On the other hand $\operatorname{Locus}(V) \subseteq \operatorname{Locus}(R) \subseteq E$ and thus

$$
2 n-2 \geq \operatorname{dim} \operatorname{Locus}(V)+\operatorname{dim} \operatorname{Locus}(V, 0 \rightarrow x),
$$


forcing $\operatorname{Locus}(V)=\operatorname{Locus}(V, 0 \rightarrow x)=E, \operatorname{dim} V=2 n-1$ and $-K_{X} \cdot C=$ $n-1$.

Let $f^{\prime}: \mathbb{P}^{1} \rightarrow \Gamma \subset E$ be a curve of the family which intersects the smooth locus of $E$; since every element of $\operatorname{Hom}_{\left[f^{\prime}\right]}\left(\mathbb{P}^{1}, E\right)$ is also an element of $\operatorname{Hom}_{\left[f^{\prime}\right]}\left(\mathbb{P}^{1}, X\right)$ and $\operatorname{Locus}(V)=E$ we can take an irreducible component of $\operatorname{Hom}_{\left[f^{\prime}\right]}\left(\mathbb{P}^{1}, E\right)$, call it $W$, which is contained in $V$; this implies that $2 n-1=\operatorname{dim} V \geq \operatorname{dim} W$.

On the other hand, since $E$ is a locally complete intersection, being a divisor in a smooth variety, we can apply [Kol96, Theorem II.1.3] which gives $\operatorname{dim} W \geq-K_{E} \cdot \Gamma+n-1$; combining the two inequalities we get

$$
-K_{E} \cdot \Gamma \leq n
$$

Recalling that E.R<0, by the adjunction formula $K_{E}=\left(K_{X}+E\right)_{\mid E}$, we have that $E \cdot \Gamma=-1$ and $-K_{E} \cdot \Gamma=n$.

Note that we can apply [Kol96, IV.3.13.3] to the scheme $E$ and the family $V$; in fact for a generic point $x \in E$ we have seen that $\operatorname{Locus}(V, 0 \rightarrow$ $x)=E$.

Thus we obtain that the Picard number of $E$ is one and $-K_{E}$ is numerically equivalent to $-n E_{E}$. Let $\pi: \widetilde{E} \rightarrow E$ be the normalization of $E$; we have that

$$
-K_{\widetilde{E}}=-\pi^{*} K_{E}+(\text { conductor of } \pi)
$$

where the conductor of $\pi$ is an effective divisor which is zero iff $\pi$ is an isomorphism ([Har77, Ex. III.7.2]). Thus

$$
-K_{\widetilde{E}}\left(\pi^{*}\left(-E_{E}\right)\right)^{n-2} \geq-K_{E}\left(-E_{E}\right)^{n-2}=n\left(-E_{E}\right)^{n-1}=n\left(\pi^{*}\left(-E_{E}\right)\right)^{n-1} .
$$

We lift the curves of $V$ to $\widetilde{E}$ and we apply [KK00, Lemma 8], see 2.3, which gives $\widetilde{E}=\mathbb{P}^{n-1}$ and $\pi^{*}\left(-E_{E}\right)=\mathcal{O}_{\mathbb{P}}(1)$. Since

$$
-K_{\widetilde{E}}=-\pi^{*} K_{E}+(\text { conductor of } \pi)=n \pi^{*}\left(-E_{E}\right)+(\text { conductor of } \pi)
$$

the conductor of $\pi$ is zero, i.e. $\pi$ is an isomorphism.

To conclude the proof of the theorem we apply the following

Theorem 3.1. (Castelnuovo) Let $E \subset X$ be a Cartier divisor in a smooth projective variety $X$ with $E \cong \mathbb{P}^{n-1}$ and $E_{E}=\mathcal{O}_{\mathbb{P}}(-1)$; then there exists a morphism $\varphi: X \rightarrow X^{\prime}$ into a smooth projective variety $X^{\prime}$ which is the blow-up of $X^{\prime}$ at a point such that $E$ is the exceptional divisor. 
The theorem was stated and proved in the case $\operatorname{dim} X=2$ by Castelnuovo; the same proof (see for instance [Har77, V.5.7]) applies in the general case.

\section{§4. An application: the first reduction}

An immediate application of the main theorem gives the following fundamental result for the so called adjunction theory; in the case Char $(k)=0$ the theorem was proved in [ABW92].

THEOREM 4.1. Let $X$ be an $n$-dimensional smooth variety over an algebraically closed field $k$ with $n \geq 3$; let also $\mathcal{E}$ be an ample vector bundle on $X$ of rank $(n-1)$ such that $K_{X}+\operatorname{det} \mathcal{E}$ is nef and big, but not ample. Then there exists a smooth variety $X^{\prime}$ and a morphism $\varphi: X \rightarrow X^{\prime}$ expressing $X$ as blow-up of $X^{\prime}$ at a finite set of points $B$ and an ample vector bundle $\mathcal{E}^{\prime}$ on $X^{\prime}$ such that $\mathcal{E} \otimes\left(\left[\varphi^{-1}(B)\right]\right)=\varphi^{*} \mathcal{E}^{\prime}$ and $K_{X^{\prime}}+\operatorname{det} \mathcal{E}^{\prime}$ is ample. The pair $\left(X^{\prime}, \mathcal{E}^{\prime}\right)$ is called the first reduction of $(X, \mathcal{E})$.

Proof. By Mori's cone theorem there exists an extremal face $F \subset$ $\overline{N E(X)}_{K_{X}<0}$ on which $K_{X}+\operatorname{det} \mathcal{E}$ is trivial; in other words $K_{X}+\operatorname{det} \mathcal{E}$ is the supporting divisor of a face $F$. By assumption every extremal ray in $F$ is non nef and has length $\geq(n-1)$ (use Propositions 2.2 and 2.1), hence we can apply the above theorem to each of these rays.

If $R_{i}$ and $R_{j}$ are two different rays in $F$ then the respective Loci, $E_{i}$ and $E_{j}$, are disjoint. In fact, assume the contrary; then, by Serre's inequality, we have

$$
\operatorname{dim}\left(E_{i} \cap E_{j}\right) \geq \operatorname{dim} E_{i}+\operatorname{dim} E_{j}-n \geq n-2 \geq 1
$$

so that the intersection of $E_{i}$ and $E_{j}$ would contain a curve $B$ and this is a contradiction.

Finally let $\mathcal{E}^{\prime}$ be a rank $n-1$ vector bundle such that $\mathcal{E} \otimes\left(\sum E_{i}\right)=\varphi^{*} \mathcal{E}^{\prime}$; it is easy to check that $\mathcal{E}^{\prime}$ and $K_{X^{\prime}}+\operatorname{det} \mathcal{E}^{\prime}$ are ample, using the Kleiman ampleness criterium, and that $K_{X}+\operatorname{det} \mathcal{E}=\varphi^{*}\left(K_{X^{\prime}}+\operatorname{det} \mathcal{E}^{\prime}\right)$.

This result implies the one stated in the introduction taking $\mathcal{E}=$ $\oplus^{(n-1)} L$ and using the following simple result:

LEMMA 4.2. Let $X$ be an $n$-dimensional smooth variety over an algebraically closed field $k$ with $n \geq 3$; let also $\mathcal{E}$ be an ample vector bundle on $X$ of rank $\geq(n-1)$. If there is a non nef ray in the cone $\overline{N E(X)}\left(K_{X}+\operatorname{det} \mathcal{E}\right) \leq 0$ then, with the only exception given by the blow-up of $\mathbb{P}^{3}$ in one point, 
$\pi: B l_{x} \mathbb{P}^{3} \rightarrow \mathbb{P}^{3}$, and $\mathcal{E}=\oplus^{2}\left(\pi^{*}\left(\mathcal{O}_{\mathbb{P}^{3}}(2)\right)-\left[\pi^{-1}(x)\right]\right)$, all rays in this cone are non nef.

Proof. Assume we have a non nef ray $R_{1}$ and a nef one $R_{2}$ as in the lemma. The ray $R_{1}$ satisfies the assumptions of Theorem (1.1), thus in particular $\operatorname{Locus}\left(R_{1}\right)=\mathbb{P}^{n-1}$. Let $V_{2} \subset \operatorname{Hom}_{\text {bir }}\left(\mathbb{P}^{1}, X\right)$ be the family associated to $R_{2}$ described in section 2 and let $x \in \operatorname{Locus}\left(R_{1}\right)$ be a general point; since by $(2.1) \operatorname{dim} \operatorname{Locus}\left(V_{2}, 0 \rightarrow x\right) \geq n-2$ we have, by Serre's inequality, that the intersection $\operatorname{Locus}\left(V_{2}, 0 \rightarrow x\right) \cap \operatorname{Locus}\left(R_{1}\right)$ contains a curve, unless $n=3$ and $\operatorname{dim} \operatorname{Locus}\left(V_{2}, 0 \rightarrow x\right)=1$.

In the general case this is a contradiction; in the special one, since extremal rays and their contractions are known in all characteristic in dimension 3 (see [Kol91]), we have that the contraction of the extremal ray $R_{2}, \varphi: X \rightarrow Z$ gives $X$ a structure of a $\mathbb{P}^{1}$ bundle of which $\operatorname{Locus}\left(R_{1}\right)=\mathbb{P}^{2}$ is a (multi)section. Let $\pi: X \rightarrow X^{\prime}$ be the contraction of $R_{1}$ and $x^{\prime}=$ $\pi\left(\operatorname{Locus}\left(R_{1}\right)\right)$; the images of the fibers of $\varphi$ via $\pi$ are irreducible rational curves passing through $x^{\prime}$ and covering $X^{\prime}$, hence we can apply [Kol96, IV.3.13.3] to deduce that the Picard number of $X^{\prime}$ is one. By the blow up formula $-K_{X^{\prime}}$ is ample so $X^{\prime}$ is Fano; then one can compute that the length of the unique ray of $X^{\prime}$ is 4 and therefore that $X^{\prime} \simeq \mathbb{P}^{3}$, by the classification of Fano threefolds [SB97].

Remark 4.3. The description of the nef rays in $\overline{N E(X)}\left(K_{X}+(n-1) L\right) \leq 0$ (or, more generally, in $\left.\overline{N E(X)}\left(K_{X}+\operatorname{det} \mathcal{E}\right) \leq 0\right)$ is well understood in characteristic zero ([Fuj87] and [ABW92]). In positive characteristic the description of such rays was recently given if $l(R)>(n-1)$, see [KK00].

If $l(R)=n-1$ we cannot at the moment say too much, the main problem being the lack of a general contraction theorem for Mori rays.

Studying the families of rational curves arising from extremal rays we can prove that, if there exists a ray of this kind, either the Picard number of $X$ is one and $-K_{X}$ is numerically equivalent to $(n-1) H$ or there exists an unsplit covering family $V$ of rational curves such that $\operatorname{dim} \operatorname{Locus}(V, 0 \rightarrow x)$ $=n-2$, for a general $x \in X$.

\section{$\S 5$. Non nef extremal rays of high length}

In this section we restrict ourselves to characteristic zero. In this case to each extremal ray $R$ is associated an extremal contraction $\varphi_{R}: X \rightarrow X^{\prime}$, that is a morphism onto a normal projective variety $X^{\prime}$ with connected fibers and such that if $C \subset X$ is a curve then $\varphi(C)$ is a point if and only 
if $[C] \in R$. Denoting by $E(\varphi)$ the exceptional locus of $\varphi$ and by $S$ an irreducible component of a (non trivial) fiber, the inequality in 2.1 can be read as

$$
\operatorname{dim} E(\varphi)+\operatorname{dim} S \geq \operatorname{dim} X+l(R)-1
$$

We first prove that the same argument in the proof of theorem (1.1) can be used to prove Conjecture (2.6) in [AW97] in the case of divisorial contractions:

THEOREM 5.1. Let $X$ be a smooth $n$-dimensional projective variety over an algebraically closed field of characteristic zero; the two following facts are equivalent:

1) There exists an extremal ray $R$ such that the contraction associated to $R$ is divisorial and the fibers have dimension $=l(R)$.

2) There exists a morphism $\varphi: X \rightarrow X^{\prime}$ into a smooth projective variety $X^{\prime}$ which is the blow-up of $X^{\prime}$ along a smooth subvariety of codimension $l(R)+1$.

Proof. We will only prove that 1) implies 2), the other implication being trivial. We observe also that it is enough to prove that there exists a line bundle $L$ on $X$ such that $L . C=1$; in fact, if this is the case, the contraction of $R$ will be supported by a divisor of the type $K_{X}+l(R) L$ with $L$ a $\varphi$-ample line bundle. Then the theorem will follow from [AW93, Theorem 4.1.iii].

Let then $R$ be an extremal ray as in 1). Since $R$ is not nef, there exists an irreducible divisor $E$ such that $E(\varphi)=\operatorname{Locus}(R)$ is contained in $E$ and $E . R<0$; let $V$ be an unsplit family of deformations of $C$. Since by the assumptions the inequality in (2.1) is an equality we have that

$$
\operatorname{dim} V=n+l(R)=n-K_{X} \cdot C .
$$

On the other hand we can think of $V$ as a family in $E$, as in the proof of 1.1 , thus, if we apply [Kol96, Theorem II.1.3], we have $\operatorname{dim} V \geq-K_{E} \cdot C+n-1$. Combining the two formulas we get

$$
-K_{E} \cdot C \leq l(R)+1
$$

Recalling that E.R $<0$ and that by the adjunction formula $K_{E}=$ $\left(K_{X}+E\right)_{\mid E}$, we have that E.C $=-1$. 
In the special case of characteristic zero we can further extend our classification of non nef extremal rays with high (but not maximal) length as in the following theorem.

THEOREM 5.2. Let $X$ be a smooth $n$-dimensional projective variety over an algebraically closed field of characteristic zero and $R=\mathbb{R}_{+}[C]$ a non nef extremal ray. Let $\varphi: X \rightarrow X^{\prime}$ be the birational elementary contraction associated to $R$ and $E=\operatorname{Exc}(\varphi)$ its exceptional locus. If $l(R)=n-2$ then one of the following cases occur:

1) $\varphi(E)$ is a point and $\left(E,-E_{E}\right) \simeq\left(\mathbb{P}^{n-1}, \mathcal{O}_{\mathbb{P}}(2)\right)$.

2) $\varphi(E)$ is a point and $\left(E,-E_{E}\right) \simeq\left(\mathbb{Q}^{n-1}, \mathcal{O}_{\mathbb{Q}}(1)\right)$, where $\mathbb{Q}^{n-1}$ is a possibly singular quadric.

3) $X^{\prime}$ is smooth and $\varphi$ is the blow-up along a smooth curve $\varphi(E) \subset X^{\prime}$.

Proof. Also here it is enough to prove that there exists a line bundle $L$ on $X$ such that $L . C=1$; then the theorem will follow from [Fuj87, Theorem 4] and [AW93, Theorem 4.1.iii].

Since $R$ is not nef, there exists an irreducible divisor $E$ such that Locus $(R)$ is contained in $E$ and $E . R<0$.

Let $V$ be the unsplit family of deformations of $C$, a minimal extremal rational curve; by the inequality in (2.1) we have that $\operatorname{Locus}(R)=E$ and $\operatorname{dim} V=\operatorname{dim} \operatorname{Locus}(V)+\operatorname{dim} \operatorname{Locus}(V, 0 \rightarrow x)+1$ is either $2 n-1$ or $2 n-2$. On the other hand we can think at $V$ as a family in $E$, as in the previous proofs. Thus, if we apply [Kol96, Theorem II.1.3], we have $\operatorname{dim} V \geq$ $-K_{E} \cdot C+n-1$. In particular we get

$$
-K_{E} \cdot C \leq n
$$

Recalling that E.R $<0$ and that by the adjunction formula $K_{E}=$ $\left(K_{X}+E\right)_{\mid E}$, we have that E.C $=-1$ or -2 .

In the first case we are done; the second one, on the other hand, can occur only if $\operatorname{dim} V=2 n-2$ and $\operatorname{dim} \operatorname{Locus}(V, 0 \rightarrow x)=n-1$. In particular we have that $\operatorname{Locus}(V, 0 \rightarrow x)=E$, so we can apply [Kol96, IV.3.13.3] to get that the Picard number of $E$ is one. Let $\pi: \widetilde{E} \rightarrow E$ be the normalization of $E$; lifting the family $V$ to $\widetilde{E}$ we obtain a new family which, together with $-\pi^{*} E_{E}$, satisfies on $\widetilde{E}$ the assumptions of [Keb00, Theorem 3.6]. This yields that $\widetilde{E} \simeq \mathbb{P}^{n-1}$ and $-\pi^{*} E_{E} \simeq \mathcal{O}_{\mathbb{P}}(2)$. Let $H_{E}$ be the ample generator of $\operatorname{Pic}(E)$; in the numerical equivalence we can write $-E_{E}=e H_{E},-K_{E}=$ $\left(\frac{n e}{2}\right) H_{E}, \quad \pi^{*} H_{E}=\mathcal{O}_{\mathbb{P}}(2 / e)$; then

$$
-K_{\widetilde{E}}=-\pi^{*} K_{E}+(\text { conductor of } \pi),
$$


hence

$$
\frac{n e}{2} \mathcal{O}_{\mathbb{P}}(2 / e)=-K_{\widetilde{E}}=\pi^{*}\left(\frac{n e}{2}\right) H_{E}+(\text { conductor of } \pi)
$$

so that the conductor is zero and $\pi$ is an isomorphism.

\section{REFERENCES}

[ABW92] M. Andreatta, E. Ballico, and J. A. Wiśniewski, Vector bundles and adjunction, Intern. J. Math., 3 (1992), 331-340.

[ABW93] - Two theorems on elementary contractions, Math. Ann., 297 (1993), 191-198.

[AW93] M. Andreatta and J. A. Wiśniewski, A note on nonvanishing and applications, Duke Math. J., 72 (1993), 739-755.

[AW97] —, A view on contraction of higher dimensional varieties, In "Algebraic Geometry - Santa Cruz 1995", Proc. Sympos. Pure Math. 62, 153-183. Amer. Math. Soc., Providence, RI, 1997.

[BS95] M. C. Beltrametti and A. J. Sommese, The adjunction theory of complex projective varieties, Exp. Math. 16, de Gruyter, Berlin, 1995.

[CE01] G. Castelnuovo and F. Enriques, Sopra alcune questioni fondamentali nella teoria delle superficie algebriche, Annali di matematica pura ed applicata, VI (1901), 165-225.

[Fuj87] T. Fujita, On polarized manifold whose adjoint bundles are not semipositive, In "Algebraic geometry, Sendai", Adv. Studies in Pure Math. 16, 167-178, Kinokuniya-North-Holland, 1987.

[Har77] R. Hartshorne, Algebraic Geometry, GTM 52, Springer-Verlag, 1977.

[Keb00] S. Kebekus, Families of singular rational curves, J. Algebraic Geom., 11 (2002), 245-256.

[KK00] Y. Kachi and J. Kollár, Characterization of $\mathbb{P}^{n}$ in arbitrary characteristic, Asian J. Math., 4 (2000), 115-122.

[Kol91] J. Kollár, Extremal rays on smooth threefolds, Ann. Sci. Ecole Norm. Sup., 24 (1991), 339-361.

[Kol96] — Rational Curves on Algebraic Varieties, Ergebnisse der Math. 32, Springer Verlag, Berlin, Heidelberg, New York, Tokyo, 1996.

[SB97] N.I. Shepherd-Barron, Fano threefolds in positive characteristic, Compositio Math., 105 (1997), 237-265.

Marco Andreatta

Dipartimento di Matematica

Università degli Studi di Trento

Via Sommarive 14

I-38100 Povo (TN)

ITALY

andreatt@science.unitn.it 
Gianluca Occhetta

Dipartimento di Matematica

Università degli Studi di Trento

Via Sommarive 14

I-38100 Povo (TN)

ITALY

occhetta@science.unitn.it 